\title{
A linear approximation for solutions of Abel-Volterra integral equations
}

\author{
Mostefa Nadir ${ }^{1}$
}

Published online: 18 May 2020

(c) The Author(s) 2020

\begin{abstract}
In this work, we present a modified linear approximation for solving the first and the second kind Abel-Volterra integral equations. This approximation was used by the author to approximate a weakly singular integral on the curve. Noting that this new technique gives a good approximation of these solutions compared with several methods in several numerical examples.
\end{abstract}

Keywords Abel-Volterra integral equations · Modified linear approximation · Weakly singular integral

Mathematics Subject Classification 45D05 - 45E05 - 45L05 - 45L10 - 65R20

\section{Introduction}

Many numerical methods are developed for solving weakly singular integral equations in particular Abel's integral equations, where in [1] the authors construct a numerical technique for solving these equations by the use of the spectral collocation method, in [2] the authors present a Method to approximate solution of the First kind Abel's integral equation using Navot's quadrature and Simpson's rule where they transform this equation to the second kind integral equation. The authors of [3] use the fractional calculus properties for solving Abel's integral equations, they approximate the functions by Chebyshev polynomials and using the collocation method to obtain the system of linear equations.

Also in [5] we find a numerical method based on the normalized Bernstein polynomials for solving singular integral equations of Abel type where the authors use the orthogonality to reduce the integral equation to a system of algebraic equations.

Communicated by José Alberto Cuminato.

Mostefa Nadir

mostefanadir@yahoo.fr

1 Department of Mathematics, University of Msila, 28000 M'Sila, Algeria 
On the other hand, the author in [7-9] presents a new method based on a linear and quadratic approximations in order to solve weakly and logarithmic integral equations.

A general Abel's integral equation of the first kind can be written as

$$
\int_{a}^{x} \frac{1}{(x-t)^{\alpha}} \varphi(t) d t=f(x), 0 \leq \alpha<1
$$

where $f(x)$ is the data with $f(a)=0$ and the function $\varphi(t)$ is the unknown function to be determined, noting that, the exact solution for $\alpha=\frac{1}{2}$ is given by

$$
\varphi(x)=\frac{\Gamma(\alpha)}{\Gamma(1-\alpha)} \frac{d}{d x} \int_{a}^{x} \frac{1}{(x-t)^{1-\alpha}} f(t) d t .
$$

On the other hand, a general Abel's integral equation of the second kind is given by

$$
\varphi(x)-\int_{a}^{x} \frac{k(x, t)}{(x-t)^{\alpha}} \varphi(t) d t=f(x), \quad 0 \leq \alpha<1 .
$$

where $k(x, t)$ is a map from $[a, b] \times[a, b]$ into $\mathbb{R}$, the unknown $\varphi(t)$ is defined on $[a, b]$. We can put the Eq. (3) in the linear functional equation form

$$
\varphi(x)-A \varphi(x)=f(x),
$$

where the operator $A$ is given by

$$
A \varphi(x)=\int_{a}^{x} \frac{k(x, t)}{(x-t)^{\alpha}} \varphi(t) d t
$$

\section{The quadrature}

Consider that $N$ is an arbitrary natural number and divide the interval $[a, b]$ into $N$ equal subintervals of $[a, b]$

$$
[a, b]=\left\{a=t_{0}<t_{1}<\ldots . .<t_{N}=b\right\},
$$

denoted $I_{1}$ to $I_{N}$, so that, we have $I_{\sigma+1}=\left[t_{\sigma}, t_{\sigma+1}\right]$, with the relation

$$
t_{\sigma}=a+\sigma \frac{l}{N}, l=b-a, \sigma=0,1,2, \ldots ., N .
$$

For an arbitrary number $\sigma=0,1,2, \ldots, N-1$, we define the spline function $S_{1}(\varphi ; t)$ depending of $\varphi, t$ and let $\sigma$ represent the linear approximation of the function density $\varphi(t)$ on the subinterval $\left[t_{\sigma}, t_{\sigma+1}\right]$ of the interval $[a, b]$. The interpolation of the density function $\varphi(t)$ with respect to the values $\varphi\left(t_{\sigma}\right)$ and $\varphi\left(t_{\sigma+1}\right)$ at the points $t_{\sigma}$ and $t_{\sigma+1}$ respectively with a linear polynomial are given by the following formula

For $t_{\sigma} \leq t \leq t_{\sigma+1}$ 


$$
S_{1}(\varphi ; t)=\frac{\left(t_{\sigma+1}-t\right)}{\left(t_{\sigma+1}-t_{\sigma}\right)} \varphi\left(t_{\sigma}\right)+\frac{\left(t-t_{\sigma}\right)}{\left(t_{\sigma+1}-t_{\sigma}\right)} \varphi\left(t_{\sigma+1}\right) .
$$

This spline function exists and is unique also called a linear interpolating polynomial. Define the function $\beta_{\sigma}(\varphi ; t, x)$ depending on $\varphi, t$ and $x$ by

$$
\beta_{\sigma}(\varphi ; t, x)=U_{\sigma}(\varphi ; t, x)-V_{\sigma}(\varphi ; t, x),
$$

where the function $U_{\sigma}(\varphi ; t, x)$ represents a modified linear interpolation of the function density $\varphi(t)$ on the subinterval $\left[t_{\sigma}, t_{\sigma+1}\right]$.

$$
U_{\sigma}(\varphi ; t, x)=\frac{\left(t_{\sigma+1}-t\right)}{\left(t_{\sigma+1}-t_{\sigma}\right)} \varphi\left(t_{\sigma}\right) \frac{(x-t)^{\alpha}}{\left(x-t_{\sigma}\right)^{\alpha}}+\frac{t-t_{\sigma}}{\left(t_{\sigma+1}-t_{\sigma}\right)} \varphi\left(t_{\sigma+1}\right) \frac{(x-t)^{\alpha}}{\left(x-t_{\sigma}\right)^{\alpha}},
$$

and the function $V_{\sigma}(\varphi ; t, x)$ is given as an approximate function of $\varphi(x)$ on the last subinterval $\left[t_{\sigma}, x\right]$ where $x=t_{\sigma+1}$ by

$$
V_{\sigma}(\varphi ; t, x)=S_{1}(\varphi ; x) \frac{(x-t)^{\alpha}}{\left(x-t_{\sigma}\right)^{\alpha}}
$$

Denote $\psi_{\sigma}(\varphi ; t, x)$ the approximation of the density $\varphi(t)$ at the point $t \in\left[t_{\sigma}, t_{\sigma+1}\right]$, and $0 \leq \sigma \leq N-1$

$$
\psi_{\sigma}(\varphi ; t, x)=\varphi(x)+\beta_{\sigma}(\varphi ; t, x)
$$

and replace this latter in the Abel integral

$$
A(x)=\int_{a}^{x} \frac{k(x, t)}{(x-t)^{\alpha}} \varphi(t) d t
$$

so that, we obtain the following approximation

$$
A_{\sigma}(\varphi ; x, t)=\sum_{\sigma=0}^{N-1} \int_{t_{\sigma}}^{t_{\sigma+1}} k(x, t) \frac{\psi_{\sigma}(\varphi ; t, x)}{(x-t)^{\alpha}} d t
$$

\section{Main results}

Theorem Let $\varphi$ be a function density continuous on $[a, b], \varphi(x) \in C([a, b])$ then, the following estimation

$$
\left|A(x)-A_{\sigma}(\varphi ; x, t)\right| \leq \frac{C}{N^{2}}
$$

holds, where $C$ is a positive constant. 


\section{Proof}

$$
\begin{aligned}
\left|A(x)-A_{\sigma}(\varphi ; t, x)\right| & =\left|\sum_{\sigma=0}^{N-1} \int_{t_{\sigma}}^{t_{\sigma+1}} k(x, t) \frac{\varphi(t)-\psi\left(\varphi_{\sigma} ; t, x\right)}{(x-t)^{\alpha}} d t\right| \\
& =\left|\begin{array}{c}
\sum_{\sigma=0}^{N-1} \int_{t_{\sigma}}^{t_{\sigma+1}} \varphi(t)-\frac{\left(t_{\sigma+1}-t\right)}{\left(t_{\sigma+1}-t_{\sigma}\right)} \varphi\left(t_{\sigma}\right) \frac{(x-t)^{\alpha}}{\left(x-t_{\sigma}\right)^{\alpha}} \\
-\frac{t-t_{\sigma}}{\left(t_{\sigma+1}-t_{\sigma}\right)} \varphi\left(t_{\sigma+1}\right) \frac{(x-t)^{\alpha}}{\left(x-t_{\sigma}\right)^{\alpha}} \times \frac{k(x, t)}{\left(x-t_{\sigma}\right)^{\alpha}} d t \\
+\sum_{\sigma=0}^{N-1} \int_{t_{\sigma}}^{t_{\sigma+1}} S_{1}(\varphi ; x) \frac{(x-t)^{\alpha}}{\left(x-t_{\sigma}\right)^{\alpha}}-\varphi(x) \times \frac{k(x, t)}{\left(x-t_{\sigma}\right)^{\alpha}} d t
\end{array}\right|
\end{aligned}
$$

or still

$$
\begin{aligned}
\left|A(x)-A_{\sigma}(\varphi ; t, x)\right| \leq\left|\begin{array}{l}
\sum_{\sigma=0}^{N-1} \int_{t_{\sigma}}^{t_{\sigma+1}} \varphi(t)-\frac{\left(t_{\sigma+1}-t\right)}{\left(t_{\sigma+1}-t_{\sigma}\right)} \varphi\left(t_{\sigma}\right) \frac{(x-t)^{\alpha}}{\left(x-t_{\sigma}\right)^{\alpha}} \\
-\frac{t-t_{\sigma}}{\left(t_{\sigma+1}-t_{\sigma}\right)} \varphi\left(t_{\sigma+1}\right) \frac{(x-t)^{\alpha}}{\left(x-t_{\sigma}\right)^{\alpha}} \times \frac{k(x, t)}{\left(x-t_{\sigma}\right)^{\alpha}} d t
\end{array}\right| \\
+\left|\sum_{\sigma=0}^{N-1} \int_{t_{\sigma}}^{t_{\sigma+1}} S_{1}(\varphi ; x) \frac{(x-t)^{\alpha}}{\left(x-t_{\sigma}\right)^{\alpha}}-\varphi(x) \times \frac{k(x, t)}{\left(x-t_{\sigma}\right)^{\alpha}} d t\right|
\end{aligned}
$$

and therefore

$$
\begin{aligned}
& \left|A(x)-A_{\sigma}(\varphi ; t, x)\right| \leq\left|\begin{array}{l}
\sum_{\sigma=0}^{N-1} \int_{t_{\sigma}}^{t_{\sigma+1}} \frac{k(x, t)}{\left(x-t_{\sigma}\right)^{\alpha}} \\
\left(\varphi(t)-\frac{\left(t_{\sigma+1}-t\right)}{\left(t_{\sigma+1}-t_{\sigma}\right)} \varphi\left(t_{\sigma}\right)-\frac{t-t_{\sigma}}{\left(t_{\sigma+1}-t_{\sigma}\right)} \varphi\left(t_{\sigma+1}\right)\right) d t
\end{array}\right| \\
& +\left|\sum_{\sigma=0}^{N-1} \int_{t_{\sigma}}^{t_{\sigma+1}} \frac{k(x, t)}{\left(x-t_{\sigma}\right)^{\alpha}}\left(S_{1}(\varphi ; x)-\varphi(x)\right) d t\right|=O\left(N^{-2}\right) .
\end{aligned}
$$

Corollary For all $\varphi(x) \in C([a, b])$, we have the standard result

$$
\left\|\varphi-\varphi_{\sigma}\right\|=O\left(N^{-2}\right)
$$

where $\varphi(x)$ is the solution of the Eq. (4)

$$
\varphi(x)-A(\varphi)=f(x)
$$

and $\varphi_{\sigma}(x)$ is the solution of the approximate equation

$$
\varphi_{\sigma}(x)-A_{\sigma}(\varphi ; t, x)=f(x) .
$$


Indeed, it is sufficient to use the theorem above.

\section{Numerical experiments}

In this section we describe some of the numerical experiments performed in solving the Abel-Volterra integral Eq. (3). In all cases, the interval is $[0,1]$ and we chose the right hand side $f(x)$ in such way that we know the exact solution. This exact solution is used only to show that the numerical solution obtained with the method is correct.

In each table, $\varphi$ represents the given exact solution of the Abel-Volterra integral equations and $\varphi_{\sigma}$ corresponds to the approximate solution of the equation produced by our linear approximation method.

Example 1 Consider the Abel-Volterra integral equation

$$
\varphi(x)-\int_{0}^{x} \frac{1}{(x-t)^{\frac{1}{2}}} \varphi(t) d t=x+\frac{2}{\pi} \sqrt{x},
$$

where the function $f(x)$ is chosen so that the solution $\varphi(x)$ is given by

$$
\varphi(x)=\frac{2}{\pi} \sqrt{x}
$$

Table 1 we present the exact solution $\varphi(x)$ and the approximate one $\varphi_{\sigma}(x)$ obtained by our approximation of the equation in the Example 1 in some arbitrary points and compared with the orthogonal Functions method [4], the error is given for $\mathrm{N}=20$.

Example 2 Consider the Abel-Volterra integral equation

$$
\varphi(x)-\int_{0}^{x} \frac{1}{(x-t)^{\frac{1}{3}}} \varphi(t) d t=x^{\frac{7}{6}},
$$

where the function $f(x)$ is chosen so that the solution $\varphi(x)$ is given by

Table 1 We present the exact solution $\varphi(x)$ and the approximate one $\varphi_{\sigma}(x)$ obtained by our approximation of the equation in the Example 1 in some arbitrary points and compared with the orthogonal Functions method [9], the error is given for $\mathrm{N}=20$

\begin{tabular}{lllll}
\hline Points of $\mathrm{t}$ & Exact sol $\varphi$ & Approx sol $\varphi_{\sigma}$ & Error & Error [4] \\
\hline $0.0000 \mathrm{e}+00$ & $0.0000 \mathrm{e}+00$ & $0.0000 \mathrm{e}+00$ & $0.0000 \mathrm{e}+00$ & $6.51 \mathrm{e}-02$ \\
$2.0000 \mathrm{e}-01$ & $2.8470 \mathrm{e}-01$ & $2.8530 \mathrm{e}-01$ & $6.0478 \mathrm{e}-04$ & $2.45 \mathrm{e}-03$ \\
$4.0000 \mathrm{e}-01$ & $4.0263 \mathrm{e}-01$ & $4.0295 \mathrm{e}-01$ & $3.1824 \mathrm{e}-04$ & $4.12 \mathrm{e}-03$ \\
$6.0000 \mathrm{e}-01$ & $4.9312 \mathrm{e}-01$ & $4.9333 \mathrm{e}-01$ & $2.0971 \mathrm{e}-04$ & $5.19 \mathrm{e}-03$ \\
$8.0000 \mathrm{e}-01$ & $5.6941 \mathrm{e}-01$ & $5.6956 \mathrm{e}-01$ & $1.5322 \mathrm{e}-04$ & $1.36 \mathrm{e}-03$ \\
$1.0000 \mathrm{e}+00$ & $6.3661 \mathrm{e}-01$ & $6.3673 \mathrm{e}-01$ & $1.1895 \mathrm{e}-04$ & \\
\hline
\end{tabular}


Table 2 We present the exact solution $\varphi(x)$ and the approximate one $\varphi_{\sigma}(x)$ obtained by our approximation of the equation in the Example 2 in some arbitrary points and compared with the approximate solution method [5], the error is given for $\mathrm{N}=20$

\begin{tabular}{lllll}
\hline Points of $\mathrm{t}$ & Exact sol $\varphi$ & Approx sol $\varphi_{\sigma}$ & Error & Error [6] \\
\hline $0.0000 \mathrm{e}+00$ & $0.0000 \mathrm{e}+00$ & $0.0000 \mathrm{e}+00$ & $0.0000 \mathrm{e}+00$ & $7.11 \mathrm{e}-03$ \\
$2.0000 \mathrm{e}-01$ & $4.0334 \mathrm{e}-01$ & $4.0477 \mathrm{e}-01$ & $1.4298 \mathrm{e}-03$ & $7.11 \mathrm{e}-03$ \\
$4.0000 \mathrm{e}-01$ & $5.7041 \mathrm{e}-01$ & $5.7130 \mathrm{e}-01$ & $8.9100 \mathrm{e}-04$ & $7.11 \mathrm{e}-03$ \\
$6.0000 \mathrm{e}-01$ & $6.9861 \mathrm{e}-01$ & $6.9924 \mathrm{e}-01$ & $6.3152 \mathrm{e}-04$ & $7.11 \mathrm{e}-03$ \\
$8.0000 \mathrm{e}-01$ & $8.0669 \mathrm{e}-01$ & $8.0716 \mathrm{e}-01$ & $4.7845 \mathrm{e}-04$ & $7.11 \mathrm{e}-03$ \\
$1.0000 \mathrm{e}+00$ & $9.0190 \mathrm{e}-01$ & $9.0228 \mathrm{e}-01$ & $3.7827 \mathrm{e}-04$ & $7.11 \mathrm{e}-03$ \\
\hline
\end{tabular}

Table 3 We present the exact solution $\varphi(x)$ and the approximate one $\varphi_{\sigma}(x)$ obtained by our approximation of the equation in the Example 3 in some arbitrary points and compared with the collocation methods [4], the error is given for $\mathrm{N}=20$

\begin{tabular}{lllll}
\hline Points of $\mathrm{t}$ & Exact sol $\varphi$ & Approx sol $\varphi_{\sigma}$ & Error & Error [5] \\
\hline $0.0000 \mathrm{e}+00$ & $1.0000 \mathrm{e}+00$ & $1.0000 \mathrm{e}+00$ & $0.0000 \mathrm{e}+00$ & $2.21 \mathrm{e}-03$ \\
$2.0000 \mathrm{e}-01$ & $4.9164 \mathrm{e}-01$ & $1.0000 \mathrm{e}+00$ & $3.2078 \mathrm{e}-03$ & $2.21 \mathrm{e}-03$ \\
$4.0000 \mathrm{e}-01$ & $3.9665 \mathrm{e}-01$ & $3.9486 \mathrm{e}-01$ & $1.7852 \mathrm{e}-03$ & $2.21 \mathrm{e}-03$ \\
$6.0000 \mathrm{e}-01$ & $3.4367 \mathrm{e}-01$ & $3.4246 \mathrm{e}-01$ & $1.2079 \mathrm{e}-03$ & $2.21 \mathrm{e}-03$ \\
$8.0000 \mathrm{e}-01$ & $3.0815 \mathrm{e}-01$ & $3.0726 \mathrm{e}-01$ & $8.9611 \mathrm{e}-04$ & $2.21 \mathrm{e}-03$ \\
$1.0000 \mathrm{e}+00$ & $2.8205 \mathrm{e}-01$ & $2.8135 \mathrm{e}-01$ & $7.0234 \mathrm{e}-04$ & $2.21 \mathrm{e}-03$
\end{tabular}

$$
\varphi(x)=\frac{7 \Gamma\left(\frac{1}{6}\right)}{18 \sqrt{\pi} \Gamma\left(\frac{2}{3}\right)} \sqrt{x} .
$$

Table 2 we present the exact solution $\varphi(x)$ and the approximate one $\varphi_{\sigma}(x)$ obtained by our approximation of the equation in the Example 2 in some arbitrary points and compared with the approximate solution method [6], the error is given for $\mathrm{N}=20$.

Example 3 Consider the Abel-Volterra integral equation

$$
\varphi(x)-\int_{0}^{x} \frac{1}{\sqrt{(x-t)}} \varphi(t) d t=1,
$$

where the function $f(x)$ is chosen so that the solution $\varphi(x)$ is given by

$$
\varphi(x)=\exp (\pi x) \operatorname{erf}(\sqrt{\pi x}) .
$$

Table 3 we present the exact solution $\varphi(x)$ and the approximate one $\varphi_{\sigma}(x)$ obtained by our approximation of the equation in the Example 3 in some arbitrary points and compared with the collocation methods [5], the error is given for $\mathrm{N}=20$.

Example 4 Consider the Abel-Volterra integral equation 
Table 4 We present the exact solution $\varphi(x)$ and the approximate one $\varphi_{\sigma}(x)$ obtained by our approximation of the equation in the Example 4 in some arbitrary points and compared with the expansion method [10], the error is given for $\mathrm{N}=20$

Table 5 We present the exact solution $\varphi(x)$ and the approximate one $\varphi_{\sigma}(x)$ obtained by our approximation of the equation in the Example 5 in some arbitrary points, the error is given for $\mathrm{N}=20$

\begin{tabular}{lllll}
\hline Points of $\mathrm{t}$ & Exact sol $\varphi$ & Approx sol $\varphi_{\sigma}$ & Error & Error [10] \\
\hline $0.0000 \mathrm{e}+00$ & $1.0000 \mathrm{e}+00$ & $1.0000 \mathrm{e}+00$ & $0.0000 \mathrm{e}+00$ & $6.00 \mathrm{e}-04$ \\
$2.0000 \mathrm{e}-01$ & $9.1287 \mathrm{e}-01$ & $9.1302 \mathrm{e}-01$ & $1.5351 \mathrm{e}-04$ & $1.00 \mathrm{e}-03$ \\
$4.0000 \mathrm{e}-01$ & $8.4515 \mathrm{e}-01$ & $8.4526 \mathrm{e}-01$ & $1.0881 \mathrm{e}-04$ & $4.00 \mathrm{e}-04$ \\
$6.0000 \mathrm{e}-01$ & $7.9056 \mathrm{e}-01$ & $7.9064 \mathrm{e}-01$ & $7.9324 \mathrm{e}-05$ & $3.00 \mathrm{e}-04$ \\
$8.0000 \mathrm{e}-01$ & $7.4535 \mathrm{e}-01$ & $7.4541 \mathrm{e}-01$ & $5.9094 \mathrm{e}-05$ & $9.00 \mathrm{e}-04$ \\
$1.0000 \mathrm{e}+00$ & $7.0710 \mathrm{e}-01$ & $7.0715 \mathrm{e}-01$ & $4.4691 \mathrm{e}-05$ & \\
\hline
\end{tabular}

\begin{tabular}{llll}
\hline Points of $\mathrm{t}$ & Exact sol $\varphi$ & Approx sol $\varphi_{\sigma}$ & Error \\
\hline $0.0000 \mathrm{e}+00$ & $2.0000 \mathrm{e}+00$ & $2.0000 \mathrm{e}+00$ & $0.0000 \mathrm{e}+00$ \\
$2.0000 \mathrm{e}-01$ & $2.2000 \mathrm{e}+00$ & $2.1980 \mathrm{e}+00$ & $1.9820 \mathrm{e}-03$ \\
$4.0000 \mathrm{e}-01$ & $2.4000 \mathrm{e}+00$ & $2.3982 \mathrm{e}+00$ & $1.7221 \mathrm{e}-03$ \\
$6.0000 \mathrm{e}-01$ & $2.6000 \mathrm{e}+00$ & $2.5984 \mathrm{e}+00$ & $1.5849 \mathrm{e}-03$ \\
$8.0000 \mathrm{e}-01$ & $2.8000 \mathrm{e}+00$ & $2.7985 \mathrm{e}+00$ & $1.4933 \mathrm{e}-03$ \\
$1.0000 \mathrm{e}+00$ & $3.0000 \mathrm{e}+00$ & $2.9985 \mathrm{e}+00$ & $1.4254 \mathrm{e}-03$ \\
\hline
\end{tabular}

where the function $f(x)$ is chosen so that the solution $\varphi(x)$ is given by

$$
\varphi(x)=\frac{1}{\sqrt{x+1}} .
$$

Table 4 We present the exact solution $\varphi(x)$ and the approximate one $\varphi_{\sigma}(x)$ obtained by our approximation of the equation in the Example 4 in some arbitrary points and compared with the expansion method [10], the error is given for $\mathrm{N}=20$.

Example 5 Consider the Abel-Volterra integral equation

$$
\varphi(x)-\int_{0}^{x} \frac{1}{(x-t)^{\frac{3}{4}}} \varphi(t) d t=x+2+\frac{16}{5} x^{\frac{5}{4}}+8 x^{\frac{1}{4}},
$$

where the function $f(x)$ is chosen so that the solution $\varphi(x)$ is given by

$$
\varphi(x)=x+2 .
$$

Table 5 we present the exact solution $\varphi(x)$ and the approximate one $\varphi_{\sigma}(x)$ obtained by our approximation of the equation in the Example 5 in some arbitrary points, the error is given for $\mathrm{N}=20$. 
Table 6 We present the exact solution $\varphi(x)$ and the approximate one $\varphi_{\sigma}(x)$ obtained by our approximation of the equation in the Example 6 in some arbitrary points, the error is given for $\mathrm{N}=20$

\begin{tabular}{llll}
\hline Points of $\mathrm{t}$ & Exact sol $\varphi$ & Approx sol $\varphi_{\sigma}$ & Error \\
\hline $0.0000 \mathrm{e}+00$ & $0.0000 \mathrm{e}+00$ & $0.0000 \mathrm{e}+00$ & $0.0000 \mathrm{e}+00$ \\
$2.0000 \mathrm{e}-01$ & $4.0000 \mathrm{e}-02$ & $3.9806 \mathrm{e}-02$ & $1.9308 \mathrm{e}-04$ \\
$4.0000 \mathrm{e}-01$ & $1.6000 \mathrm{e}-01$ & $1.5965 \mathrm{e}-01$ & $3.4012 \mathrm{e}-04$ \\
$6.0000 \mathrm{e}-01$ & $3.6000 \mathrm{e}-01$ & $3.5954 \mathrm{e}-01$ & $4.5853 \mathrm{e}-04$ \\
$8.0000 \mathrm{e}-01$ & $6.4000 \mathrm{e}-01$ & $6.3944 \mathrm{e}-01$ & $5.5680 \mathrm{e}-04$ \\
$1.0000 \mathrm{e}+00$ & $1.0000 \mathrm{e}+00$ & $9.9935 \mathrm{e}-01$ & $6.4007 \mathrm{e}-04$ \\
\hline
\end{tabular}

Example 6 Consider the Abel-Volterra integral equation

$$
\varphi(x)-\int_{0}^{x} \frac{1}{(x-t)^{\frac{1}{5}}} \varphi(t) d t=x^{2}+\frac{125}{252} x^{\frac{14}{5}},
$$

where the function $f(x)$ is chosen so that the solution $\varphi(x)$ is given by

$$
\varphi(x)=x^{2} .
$$

Table 6 we present the exact solution $\varphi(x)$ and the approximate one $\varphi_{\sigma}(x)$ obtained by our approximation of the equation in the Example 6 in some arbitrary points, the error is given for $\mathrm{N}=20$.

\section{Conclusion}

In this work we present a numerical solution of the general Abel-Volterra integral equations using a new approximation of the unknown function based on the modification of the linear spline function, this approximation allows us to eliminate the singularity at the endpoint

This numerical results show that the accuracy improves with increasing of the number of subdivisions. Finally we confirm that this method lead us to better approximation of the exact solution when compared with the methods in papers $[4-6,10]$.

Open Access This article is licensed under a Creative Commons Attribution 4.0 International License, which permits use, sharing, adaptation, distribution and reproduction in any medium or format, as long as you give appropriate credit to the original author(s) and the source, provide a link to the Creative Commons licence, and indicate if changes were made. The images or other third party material in this article are included in the article's Creative Commons licence, unless indicated otherwise in a credit line to the material. If material is not included in the article's Creative Commons licence and your intended use is not permitted by statutory regulation or exceeds the permitted use, you will need to obtain permission directly from the copyright holder. To view a copy of this licence, visit http://creativecommons.org/licen ses/by/4.0/. 


\section{References}

1. Abdelkawy, M.A., Ezz-Eldien, S.S., Amin, A.Z.M.: A Jacobi spectral collocation scheme for solving Abel's integral equations. Prog. Fract. Differ. Appl. 1(3), 187-200 (2015)

2. Araghi, M.A.F., Yazdani, S.: A method to approximate solution of the first kind abel integral equation using Navot's quadrature and Simpson's rule. Int. J. Ind. Math. 1(1), 1-11 (2009)

3. Avazzadeh, Z., Shafiee, B., Loghmani, G.B.: Fractional calculus for solving Abel's integral equations using chebyshev polynomials. Appl. Math. Sci. 5(45), 2207-2216 (2011)

4. Derilia, H., Sohrabib, S.: Numerical solution of singular integral equations using orthogonal functions. Math. Sci. 2(3), 261-272 (2008)

5. Diogo, T., McKee, S., Tang, T.: Collocation methods for second-kind Volterra integral equations with weakly singular kernels. Proc. R. Soc. Edinb. Sect. A Math. 124(2), 199-210 (1994)

6. Huang, Li, Huang, Yong, Li, Xian-Fang: Approximate solution of Abel integral equation. Comput. Math. Appl. 56, 1748-1757 (2008)

7. Nadir, M.: A new technical for solving a weakly singular integro-differential equations. J. Taibah Univ. Sci. 11, 229-232 (2016)

8. Nadir, M.: A modified linear approximation for weakly singular integrals. J. Theor. Appl. Comput. Sci. 9(4), 19-25 (2015)

9. Nadir, M.: Adapted quadratic approximation for logarithmic kernel integrals. Fasciculi mathematici 49(1), 75-85 (2012)

10. Shahsavaran, A., Babolian, E.: Numerical implementation of an expansion method for linear Volterra integral equations of the second kind with weakly singular kernels. Int. J. Appl. Math. Comput. 3(1), 1-8 (2011)

Publisher's Note Springer Nature remains neutral with regard to jurisdictional claims in published maps and institutional affiliations. 